Original paper

\title{
Dosimetric optimization of nuclear medicine therapy based on the Council Directive 2013/59/EURATOM and the Italian law N. 101/2020. Position paper and recommendations by the Italian National Associations of Medical Physics (AIFM) and Nuclear Medicine (AIMN)
}

\author{
Carlo Chiesa $^{\mathrm{a}, *, 1}$, Lidia Strigari ${ }^{\mathrm{b}, 1}$, Massimiliano Pacilio ${ }^{c}$, Elisa Richetta ${ }^{\mathrm{d}}$, Vittorio Cannatà ${ }^{\mathrm{e}}$, \\ Michele Stasi $^{\mathrm{d}, 3}$, Maria Cristina Marzola ${ }^{\mathrm{f}}$, Orazio Schillaci ${ }^{\mathrm{g}, \mathrm{h}, 4}$, Oreste Bagni ${ }^{\mathrm{i}, 2}$, \\ Marco Maccauro ${ }^{\text {a, } 2}$ \\ ${ }^{a}$ Nuclear Medicine, Foundation IRCCS Istituto Nazionale Tumori, Milan, Italy \\ ${ }^{\mathrm{b}}$ Director of Medical Physics, IRCCS Azienda Ospedaliero-Universitaria di Bologna, Bologna, Italy \\ ${ }^{\mathrm{c}}$ Director of Medical Physics, Azienda Ospedaliero-Universitaria Policlinico Umberto I, Rome, Italy \\ ${ }^{\mathrm{d}}$ Medical Physics, Azienda Ospedaliera Ordine Mauriziano, Turin, Italy \\ e Director of Medical Physics Unit, Medical Physics Unit, Bambino Gesù Children's Hospital, IRCCS, Rome, Italy \\ ${ }^{\mathrm{f}}$ Department of Nuclear Medicine PET/CT Centre, S. Maria della Misericordia Hospital, Rovigo, Italy \\ ${ }^{\mathrm{g}}$ Dean of University Tor Vergata, Rome, Italy \\ ${ }^{\mathrm{h}}$ IRCCS Neuromed, Pozzilli, Italy \\ ${ }^{\text {i }}$ Director of Nuclear Medicine, S. Maria Goretti Hospital, Latina, Italy
}

\section{A R T I C L E I N F O}

\section{Keywords:}

Nuclear medicine therapy

Dosimetry

Optimization

Standardized, non-standardized terapies

\begin{abstract}
A B S T R A C T
This recommendation by the Italian Associations of Nuclear Medicine (AIMN) and Medical Physics (AIFM) focuses on the dosimetric optimization of Nuclear Medicine Therapy (NMT) as clearly requested by the article 56 of the EURATOM Directive 2013/59 and its consequent implementation in article 158 in the Italian Law $\mathrm{n}$. 101/ 2020.

However, this statement must deal with scientific and methodological limits that still exist and, above all, with the currently available limited resources. This paper addresses these specific issues. It distinguishes among many possible kinds of NMT. For each type, dosimetric optimization is recommended or considered optional, according to the general criteria adopted in any human choice, i.e. a check of technical feasibility first, followed by a cost/ benefit argument. The classification of therapies as standardized or non-standardized is presented. This is based on the complexity of the type of pathology, on the variability of the treatment outcome, and on the risks involved.

According to the present document, which was officially delivered to Italian Health Ministry as necessary interpretation of the law, a therapeutic team can, in science and consciousness, overcome the indications of posology, to optimize and tailoring a treatment with dosimetry, on the basis of published national or international data or guidelines, without need of an Ethics Committee approval. Data collected in this way will provide additional evidence about optimal dosimetric reference values.

As conclusion, a formal appeal is made to the European and National regulatory agencies for pharmaceuticals to obtain the official acknowledgment of this principle.
\end{abstract}

\footnotetext{
* Corresponding author.

E-mail address: Carlo.chiesa@istitutotumori.mi.it (C. Chiesa).

1 Carlo Chiesa and Lidia Strigari are both first author of this paper. They were both members of the EANM dosimetry committee.

2 Oreste Bagni and Marco Maccauro are both last author of this paper. Dr Maccauro is the chair of the Therapy and Dosimetry committee of AIMN.

${ }^{3}$ Prof. Michele Stasi was the President of the Italian Association of Medical Physics (AIFM).

${ }^{4}$ Prof. Orazio Schillaci is the President of the Italian Association of Nuclear Medicine (AIMN).
} 


\section{Introduction}

The present consensus paper consists of the minimal recommendations by the Italian Associations of Nuclear Medicine (AIMN) and Medical Physics (AIFM) (hereinafter referred to as "the two Associations"), regarding the optimization of Nuclear Medicine Therapy (NMT) requested by the article 56 of the Council Directive 2013/59/ EURATOM about Basic Safety Standard [1] (hereinafter referred to as "the Directive") and by its consequent implementation in the Italian Law $\mathrm{n}$. 101/2020 (hereinafter referred to as "the Italian law") [2].

"Minimal recommendation" means that for the implementation of dosimetry in clinical practice we considered technical resources generally available in the routine of an Italian nuclear medicine therapy unit. Centres that are equipped with particularly advanced resources/technologies and trained staffs can/should increase the number and quality of dosimetric studies compared to what is indicated hereafter.

This document is not a methodological guideline providing detailed indications about the most appropriate dosimetric methods in the various clinical scenarios. It is rather a general management indication discussing the feasibility of dosimetry in the majority of NMT Italian centres.

The original work [3] was written in Italian language at the end of 2018 and it was delivered to the Italian Health Ministry as necessary interpretation of the Directive. The development of that work followed an established workflow for this kind of documents. Each section was discussed and written in a series of meeting of the joint AIFM-AIMN committee. Then the draft was reviewed, corrected and voted for approval by both the Executive Boards of the two Associations. Therefore, it represents the consensus between the two Associations, and not only the opinions of the writers.

It was also presented and recorded as CME lecture to the 2019 Congress of the European Association of Nuclear Medicine (EANM) [4]. After the presentation, colleagues from different countries asked for an English version of the work. These requests remarked the general difficulty in interpreting the optimization principle in NMT, in defining standardized and non-standardized therapies and the level of involvement of the medical physics expert (MPE) in NMT.

The present paper was developed to make the Italian position internationally available. It differs from the original version first for editorial reasons. The main text is limited now to the original basic arguments, concepts and to the summary table. Details pertaining to each specific kind of therapy were moved to the supplementary materials. Moreover, the criterion used to classify therapies in standardized or nonstandardized was better described.

Secondly, in the last two years, two important novelties related to this topic occurred and were included in this work.

Survey data regarding the evolution of the use of dosimetry in Italy were added.

EANM published a different interpretation of the optimization principle applied to NMT. A comparison between the two positions became necessary.

Therefore, the discussion section was enlarged to cover these facts.

In summary the core arguments, concepts and results are the same as in the original version. They are however enriched with new accessories.

\section{Basic definitions and rationale}

\section{Definitions and basic concepts}

In NMT, measuring activity (GBq) of a vial is in principle simple. Some additional calibration effort is required to obtain an acceptable accuracy level with low-energy gamma emitters or pure beta emitters. On the contrary, individual absorbed dose (Gy) calculation is more demanding, as it usually requires repeated patient scans, preliminary calibrations, dedicated software, and medical physicists for computations. However, toxicity and efficacy of a treatment depend on absorbed dose rather than on administered activity. The need of individualized dosimetry arises from intra patient differences in organ biokinetics and lesions uptake. Patients with similar clinical conditions, administered with the same activity, usually receive different absorbed doses to organs and lesions, determining different outcomes.

The definition of the Directive and of the Italian law concerning NMT state: " "radiotherapeutic": pertaining to radiotherapy, including nuclear medicine for therapeutic purposes". The two Associations interpret this definition not in the sense of an organizational or operational dependence of the NMT units and personnel from the external beam radiotherapy facilities. The definition undoubtedly enshrines that NMT belongs, first and foremost, to the field of radiotherapy, and must therefore follow indications of good radiotherapy practice, as well as the relative legislation. The definition intends to distinguish NMT from other forms of medical therapy, in particular chemotherapy and drug therapies, since the mechanism of action of NMT is mainly due to the emission of ionizing radiation, and not to chemical (pharmacological) mechanisms [5]. For this substantial reason, seen the present legislative conflict between posology and individualized dosimetry [6], the choice of activity in NMT should be performed primarily in compliance with the optimization principle of radiotherapy, according to the indications provided below, without neglecting the pharmaceutical legislation for all the other pertinent factors.

Medical devices, as radiolabelled microspheres for Selective Internal Radiation Therapy (SIRT) of liver disease neoplasm, are regulated by a different legislation, the Medical Device Directive 93/42 [7]. As a matter of fact, the legal indication regarding their dosage is indicated in the Instruction For Use (IFU), which does not fulfil the optimization principle $[8,9]$, except for the multi-compartment model indicated by Sirtex [10]. Given the same radiation mechanism, also radiolabelled microspheres should be administered primarily in compliance with the optimization principle.

\section{Optimization for all medical exposures with radiotherapeutic purpose?}

Optimization is the heart of the issues dealt with in this document and deserves several comments. Article 56 of the Directive and article 158 of the Italian law regarding optimization state that "For all medical exposures of patients for radiotherapeutic purposes, exposures of target volumes shall be individually planned and their delivery appropriately verified taking into account that doses to non-target volumes and tissues shall be as low as reasonably achievable and consistent with the intended radiotherapic purpose of the exposure ". In NMT, "optimization" means that the choice of the therapeutic activity should be based on absorbed dose values that aims at the best compromise, or balance, between efficacy (absorbed dose to target volumes) and toxicity (absorbed dose to healthy tissues).

About the statement "For all exposure....", the two Associations believe that it is not realistic to conceive a systematic optimization for all kinds of therapies in this historical phase, for various reasons:

a) technical impediments (unfeasible dosimetry) sometime linked to clinical situations. Present methodologies sometimes show instrumental limits which prevent an accurate dosimetric evaluation. The most fitting example is the impossibility of accurately measuring the target size and consequently of calculating the absorbed dose. In such cases optimization is impossible. One could cite for instance differentiated thyroid cancer (DTC) remnant after thyroidectomy, or neck lymph nodes, using the diagnostic methods currently used (ultrasound and scintigraphy), or patients with high tumoural marker levels with negative nuclear medicine imaging (micro-metastases of differentiated thyroid cancer (DTC) or neuroblastoma). Unavailability of a proper agent for predictive dosimetry is another issue. The example is ${ }^{223} \mathrm{Ra}$, which lacks of an isotope able to simulate the therapeutic lesions uptake and, above all, their retention time. 
b) unfavourable cost/benefit ratio [11]. This is the novelty of the present document and it is discussed in detail for each kind of therapy in the supplementary materials.

Cost is meant not only from the economical point of view, but as the general need of resources necessary to perform dosimetry: materials and equipment (radiopharmaceutical, SPECT/CT system, $\mathrm{PET} / \mathrm{CT}$ scanner, gamma-counter, probe); man-time (uptime for scans, human resources, and time for data collection and dosimetric calculations); dosimetric software.

Benefit is twofold: clinical, for the treated patient, and social. Indications of clinical benefit thanks to dosimetry are available in radioiodine treatment of metastatic DTC [12-14], radiopeptide treatment of neuroendocrine tumours [15] and in microspheres treatment of liver neoplasm [16] though only the last one was obtained with a multicentric prospective randomized study. Social benefit in terms of spared resources might derive from avoiding useless treatments when absorbed dose to target is predicted too low to obtain the aimed response. An indication in this direction thanks to dosimetry was already reported by Maxon et al in 1992 [17].

c) clinical and logistic impediments: in most applications, dosimetry requires repeated scans over typically one week. This procedure may be unsustainable for some patients with particularly disabling pathologies (e.g., symptomatic multiple bone metastases), or uncapable of coming back some time to the hospital as out-patients. In these cases, the therapist must record the reasons why the dosimetric practice cannot be supported by the patient and justify the method of choosing the therapeutic activity.

\section{Maximization for oncological patients with life threatening disease}

In cases of oncological patients with life-threatening disease (e.g. neuroblastoma, extensive metastatic DTC, neuroendocrine tumours, liver neoplasm), when target dosimetry is not feasible or non-reliable, accurate optimization is impossible. A different approach can be adopted, aiming to deliver the maximum tolerable activity (MTA) corresponding to the maximum tolerable absorbed dose (MTD) to non-target tissues, or to the Organs At Risk (OAR). This method is named "Maximization" $[6,18]$ and it is not new. It has been used for decades, especially with pluri-metastatic and advanced cases [6,19-22]. In these patients, the current approaches based on the administration of fixed activities times a fixed number of administrations must be improved by a dosimetric treatment planning.

The maximization method is conceptually and legally supported by the last sentence of the optimization principle: “...... and consistent with the intended radiotherapeutic purpose of the exposure". The maximization method allows, in the oncological field, to pursue the purpose of the exposure when optimization is not feasible.

Maximization can be totally inadequate from the point of patient radiation protection in other clinical situations, where an excellent percentage of successes (complete response) can be obtained by administering an activity much lower than MTA (e.g., remnant ablation in DTC, benign thyroid diseases, radio-synoviorthesis).

\section{Tissue reaction and deterministic risks}

A third factor should be included in the dilemma whether to recommend or not the dosimetric optimization (or maximization), beyond toxicity and inefficacy risks (deterministic effects): the risk of late tissue reaction (previously named stochastic effects, i.e. the induction of second tumours and hereditary effects). This phenomenon is the subject of widespread debate in the scientific literature and it cannot be discussed in this work. We considered the following position reasonable.

For oncological patients where NMT is indicated by clinical guidelines or by evidence studies, the two Associations consider stochastic risk acceptable (optimization optional), if there is evidence of a high probability of complete response or of reaching the aimed end point (e. g., bone pain palliation) in absence of toxicity to vital organs. This statement holds except for paediatric or particularly young patients, for whom optimization is a must. Typical example of this condition is the ablation of the thyroid remnant in high-risk patients.

For non-oncological pathologies, more attention should be paid to prevent tissue reaction. Dosimetric optimization should always be applied, when technically feasible with ordinary methodology. Typical examples are, on the one hand, benign thyroid pathologies, where dosimetry is technically possible and therefore recommended in the planning phase; on the other hand, in radio-synovectomy, where dosimetry currently requires very advanced techniques [23], not within the reach of most centres (technically not feasible), dosimetric planning is not recommended.

\section{Consensus statements}

Responsibility

The article 57 of the Directive ("Resposibilities") was modified in the corresponding article 159 in the Italian law, which states "All exposures are undertaken under the clinical responsibility of a medical specialist."

The two Associations believe that, for the peculiarities that distinguish nuclear medicine therapy from other forms of radiotherapy (external beams, brachytherapy), the specialist mentioned in this article is the specialist in nuclear medicine. This statement is not retroactive but holds starting from the coming into effect of the Italian law n. 101/2020, on 27 August 2020.

\section{Procedures (article 58 (d) in the Directive)}

The expression "closely involved" in non-standardized NMT means a level of involvement of MPE equivalent to what occurs in external beam radiotherapy (EBRT). This includes for instance discussing the treatment strategy with the therapist, performing individualized planning and verification, providing dose estimations, developing dosimetric protocols, calibrating instruments for quantification, overviewing activity measurements. MPE can also be involved in the measurement of exposure rate for patients to be discharged, on behalf (or having the role) of radiation protection expert, but this should not be meant as the kind of involvement requested in article 58 (d).

\section{Classification as standardized/non-standardized therapies}

In EBRT the absence of a dosimetric planning always expose a patient to the possibility of detriment or inefficacy. In NMT this is not always true, as there are situations in which the administration of fixed activity does not imply risk of toxicity or of inefficacy. These applications are therefore candidates to be classified as standardized therapies, according to article 58 (d) of the Directive.

Considering all the above aspects, the two Associations examined a list of NMTs and for each one developed an argument to indicate whether dosimetric optimization should be recommended or considered optional. Moreover, each treatment was classified as standardized or non-standardized. All results are summarized in Tables 1 and 2. Specific arguments about classifications and optimization of each therapy, and hints about dosimetric methodology are detailed in the supplementary materials online.

Shortly, therapies classified as standardized are: the treatment with ${ }^{131}$ I of remnant and metastatic neck nodes of DTC in adults, and of benign thyroid disease; therapy of bone metastases of prostate cancer treatment with ${ }^{223} \mathrm{Ra}$, bone pain palliation with ${ }^{153}$ Sm-EDTMP, of nonHodgkin's lymphoma with ${ }^{90} \mathrm{Y}$ ibritumomab-tiuxetan, and radiosynoviorthesis.

Note that all other treatments of DTC with ${ }^{131}$ I were classified as nonstandardized: distant metastases, paediatric treatments, diffuse lung 
Table 1

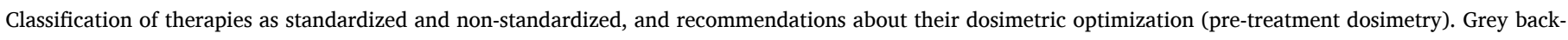
ground remarks treatments where dosimetric optimization is optional, while white background where it is recommended.

\begin{tabular}{|c|c|c|c|c|c|c|c|c|}
\hline $\begin{array}{l}\text { Kind of disease or treatment (DTC } \\
=\text { differentiated thyroid cancer) }\end{array}$ & 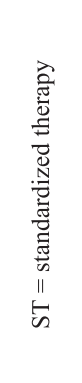 & 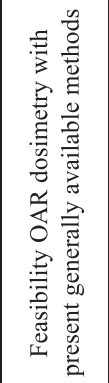 & 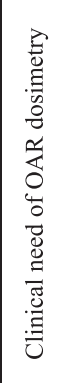 & 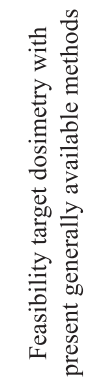 & 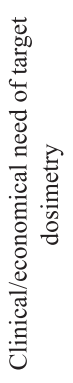 & $\begin{array}{c}\text { OPTIMIZATION WITH PRE-TREATMENT } \\
\text { DOSIMETRY }\end{array}$ & 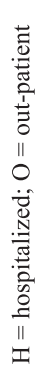 & Agent \\
\hline DTC adult remnant ablation & ST & YES & NO (1) & No & NO & Optional, on target & $\mathrm{O}$ & ${ }^{131} \mathrm{I}$ \\
\hline DTC adult neck nodes & ST & YES & NO (1) & seldom & YES & Optional, on blood and target & $\mathrm{O}$ & ${ }^{131} \mathrm{I}$ \\
\hline DTC adult distant metastases & NON ST & YES & YES & not always & YES & RECOMMENDED on blood, if possible also on target & $\mathrm{O}$ & ${ }^{131} \mathrm{I}$ \\
\hline DTC pediatric, in dyalisis, immunodepressed & NON ST & YES & YES & not always & YES & RECOMMENDED on blood, if possible also on target & $\mathrm{O}$ & ${ }^{131} \mathrm{I}$ \\
\hline DTC lung diffuse micrometastases & NON ST & YES & YES & YES & YES & RECOMMENDED on blood and on lung & $\mathrm{O}$ & ${ }^{131} \mathrm{I}$ \\
\hline Liver radioembolization with ${ }^{90} \mathrm{Y}$ microspheres & NON ST & YES & YES & YES & YES & RECOMMENDED on tumours, non-tumour and lung & $\mathrm{H}$ & ${ }^{99}{ }^{99} \mathrm{Tc}-\mathrm{MAA}$ \\
\hline Liver radioembolization with ${ }^{166} \mathrm{Ho}$ microspheres & NON ST & YES & YES & YES & YES & RECOMMENDED on tumours, non-tumour and lung & $\mathrm{H}$ & ${ }^{99 \mathrm{~m}} \mathrm{Tc}-\mathrm{MAA}$, better ${ }^{166} \mathrm{Ho}(2)$ \\
\hline mIBG pediatric & NON ST & YES & YES & not always & YES & RECOMMENDED on whole body, if possible also for target & $\mathrm{O}$ & ${ }^{131} \mathrm{I} \mathrm{mIBG}$ \\
\hline mIBG adult & NON ST & YES & NO (3) & not always & YES & Optional on whole body and on target & $\mathrm{O}$ & ${ }^{131} \mathrm{I} \mathrm{mIBG}$ \\
\hline${ }^{177}$ Lu somatostatine analogs & NON ST & YES $(*)$ & YES & YES $(*)$ & YES & RECOMMENDED $\left({ }^{*}\right)$ peri-therapeutically at 1 st administration & $\mathrm{O}$ & ${ }^{177}$ Lu-somatostine analog \\
\hline${ }^{90} \mathrm{Y}$ somatostatine analogs & NON ST & NO with ${ }^{90} \mathrm{Y}$ & YES & NO with ${ }^{90} \mathrm{Y}$ & YES & RECOMMENDED $\left({ }^{*}\right)$ peri-therapeutically at 1 st ${ }^{177} \mathrm{Lu}$ administration & $\mathrm{O}$ & - \\
\hline${ }^{223} \mathrm{Ra}$ & ST & NO & NO (4) & YES $(*)$ & YES & Optional, on target - $\left(^{*}\right)$ peri-therapy after 1st administration, 3rd \& 5th & $\mathrm{O}$ & - \\
\hline Hyperthyroidism Graves & ST & YES & NO & YES & YES & RECOMMENDED on target & $\mathrm{O}$ & ${ }^{131} \mathrm{I}$ \\
\hline Hyperthyroidism nodules & ST & YES & NO & YES & YES & RECOMMENDED on nodules and contralateral lobe & $\mathrm{O}$ & ${ }^{131} \mathrm{I}$ or ${ }^{123} \mathrm{I}$ \\
\hline non Hodgkin's lymphoma with ${ }^{90} \mathrm{Y}$ Zevalin $\circledast$ & ST & YES & NO (5) & No & NO & Optional, on OAR (red marrow, liver) & $\mathrm{O}$ & ${ }^{111}$ In-ibritumomab tiuxetan \\
\hline${ }^{153}$ Sm EDTMP Lexidronam (Quadramet®) & ST & YES & YES & YES & NO & RECOMMENDED on red marrow ( $2 \mathrm{WB}$ counts) & $\mathrm{O}$ & ${ }^{99 \mathrm{~m}} \mathrm{Tc}$-metyl-diphosphonate \\
\hline Radiosynoviorthesis & ST & NO & NO & NO & NO & Optional - not feasible with simple methods & $\mathrm{O}$ & various \\
\hline Experimental therapies & NON ST & to be defined & YES & to be defined & YES & RECOMMENDED on OAR and TARGET. Methods to be defined & - & - \\
\hline PSMA & NON ST & YES & YES & YES & YES & RECOMMENDED on OAR and TARGET. Methods to be defined & - & - \\
\hline
\end{tabular}

OAR $=$ organ at risk.

(1) Except particular cases described.

(2) Scout dose.

(3) Optional only if activity $<11$ GBq.

(4) Optional only if standard posology is used.

(5) No clinical need except rare cases.

metastases, particular cases (dialyzed and immuno-depressed patients). Radioembolization, radiopeptide therapy, ${ }^{131}$ I-mIBG, agents direct to PSMA, experimental therapies were also classified as non-standardized.

\section{Recommendations of optimization}

Optimization with pre-treatment dosimetry (Table 1) was indicated as optional in remnant ablation and neck nodes treatment in DTC, ${ }^{131} \mathrm{I}$ mIBG in adults, ${ }^{223} \mathrm{Ra}$, ${ }^{90} \mathrm{Y}$-ibritumomab tiuxetan, and radiosynoviorthesis. In all the other nuclear medicine treatment, optimization through pre-treatment dosimetry is recommended. For radiopeptides, peri-treatment dosimetry after the first administration of ${ }^{177} \mathrm{Lu}$ labelled radiopeptide fulfils the optimization recommendation about the whole course of treatments.

Dosimetric verification with peri-treatment dosimetry (Table 2) was indicated as optional in remnant ablation and neck nodes treatment in DTC, ${ }^{90} \mathrm{Y}$ somatostatin analogs, ${ }^{223} \mathrm{Ra}$, benign thyroid diseases, ${ }^{90} \mathrm{Y}$ ibritumomab-tiuxetan, and radio-synov-iorthesis. In all the other nuclear medicine treatment, dosimetric verification through peri-treatment dosimetry is recommended.

\section{Italian National surveys about dosimetry implementation}

We observed the evolution of the number of Italian centres performing dosimetry on a regular or almost regular basis according to three surveys, relative to years 2007, 2015 and to 2019 (Table 3). A visual comparison between the two latest major survey data is offered in Fig. 1. The three surveys were accomplished with slightly different questions, and with increasing level of accuracy along time. Data of
2007 were obtained through the circulation of a questionnaire on the mailing list of the Italian Internal Dosimetry Group. In 2020 a more complete questionnaire was developed by the Authors and distributed to the two mailing lists of the two Associations. Furthermore, therapy centres were repeatedly phoned until data of interest were obtained. Data of 2015 were extracted from the online supplementary materials of EANM publication by Sjögreen-Gleisner et al. [24]. The number of answering centres also increased, providing increased reliability.

In 2019, 47 nuclear medicine units with isolation rooms (for a total of 225 beds) were active in Italy (corresponding to $3.7 \mathrm{bed} / \mathrm{million}$ population). A good answering percentage was achieved with data from 43 centres (92\%) (corresponding to 205 beds). We obtained 18 additional answers from centres performing therapy without isolation rooms on out-patient basis (hyperthyroidism, hepatic radioembolization, ${ }^{223}$ Ra treatments).

In the last 4 years (2015-2019), the percentual use of pre-treatment dosimetric optimization (table 3 ) increased in radioembolization (from $18 / 21=86 \%$ to $25 / 26=96 \%$ ), in hyperthyroidism therapy (from $17 /$ $45=38 \%$ to $33 / 51=65 \%)$ and was constant in ${ }^{177}$ Lu-DOTATATE $(1 / 4$ $=7 / 28=25 \%$ used to optimize, despite the fixed posology). Conversely, DTC and ${ }^{131}$ I-mIBG (17\%), results are less encouraging, with a slight increase in the former (from $13 \%$ to $23 \%$ for distant metastases) and an apparent decrease in the latter (from about $30 \%$ to $17 \%$ ). The absolute numbers (Fig. 1) are approximately constant for DTC, mIBG and ${ }^{223} \mathrm{Ra}$, while showed a noticeable increase in ${ }^{177}$ Lu-DOTATATE, radioembolization and hyperthyroidism therapy.

The percentual use of dosimetry for treatment verification doubled in somatostatine analogs (from $25 \%$ to $53 \%$ ), increased in radioembolization (from $40 \%$ to $55 \%$ to $65 \%$ ), it was constantly low in hyperthyroidism (4\% and 6\%), and apparently decreased in DTC (from 
Table 2

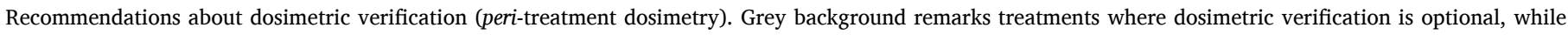
white background where it is recommended.

\begin{tabular}{|c|c|c|c|c|c|c|c|c|}
\hline $\begin{array}{l}\text { Kind of disease or treatment (DTC } \\
=\text { differentiated thyroid cancer) }\end{array}$ & 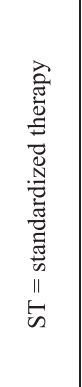 & 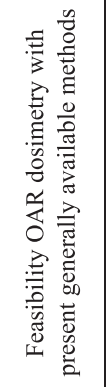 & 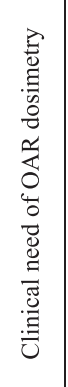 & 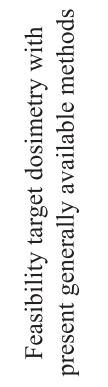 & 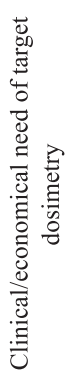 & $\begin{array}{c}\text { VERIFICATION WITH PERI-TREATMENT } \\
\text { DOSIMETRY }\end{array}$ & 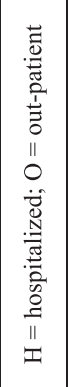 & Agent \\
\hline DTC adult remnant ablation & ST & YES & NO (1) & NO & NO & Optional, on target & $\mathrm{H}$ & ${ }^{131} \mathrm{I}$ \\
\hline DTC adult neck nodes & ST & YES & NO (1) & seldom & YES & Optional, on blood and target & $\mathrm{H}$ & ${ }^{131} \mathrm{I}$ \\
\hline DTC adult distant metastases & NON ST & YES & YES & not always & YES & RECOMMENDED on blood, if possible also on target & $\mathrm{H}$ & ${ }^{131} \mathrm{I}$ \\
\hline DTC pediatric, in dyalisis, immunodepressed & NON ST & YES & YES & not always & YES & RECOMMENDED on blood, if possible also on target & $\mathrm{H}$ & ${ }^{131} \mathrm{I}$ \\
\hline DTC lung diffuse micrometastases & NON ST & YES & YES & YES & YES & RECOMMENDED on blood and on lung & $\mathrm{H}$ & ${ }^{131} \mathrm{I}$ \\
\hline Liver radioembolization with ${ }^{90} \mathrm{Y}$ microspheres & NON ST & YES & YES & YES & YES & RECOMMENDED on tumours, non-tumour and lung & $\mathrm{H}$ & ${ }^{90} \mathrm{Y}$-microsphere PET \\
\hline Liver radioembolization with ${ }^{166} \mathrm{Ho}$ microspheres & NON ST & YES & YES & YES & YES & RECOMMENDED on tumours, non-tumour and lung & $\mathrm{H}$ & ${ }^{166} \mathrm{Ho}$ microsphere SPECT or MRI \\
\hline mIBG pediatric & NON ST & YES & YES & not always & YES & $\begin{array}{l}\text { RECOMMENDED on whole body (predictive for the next treatment), if } \\
\text { possible also on target }\end{array}$ & $\mathrm{H}$ & ${ }^{131} \mathrm{I}$ \\
\hline mIBG adult & NON ST & YES & YES & not always & YES & $\begin{array}{l}\text { RECOMMENDED on whole body (predictive for the next treatment), if } \\
\text { possible also on target }\end{array}$ & $\mathrm{H}$ & ${ }^{131} \mathrm{I}$ \\
\hline${ }^{177}$ Lu somatostatine analogs & NON ST & YES & YES & YES & YES & RECOMMENDED on OAR and target (predictive for the next treatment) & $\mathrm{O}$ & ${ }^{177} \mathrm{Lu}$-somatostine analog \\
\hline${ }^{90} \mathrm{Y}$ somatostatine analogs & NON ST & NO & YES & NO & YES & Non feasible with ${ }^{90} \mathrm{Y}$ & - & - \\
\hline${ }^{223} \mathrm{Ra}$ & ST & NO & NO (4) & not always & YES & Optional, on target - peri-therapy after 1st administration, 3rd \& 5th & $\mathrm{O}$ & ${ }^{223} \mathrm{Ra}$ \\
\hline Hyperthyroidism Graves & ST & YES & NO & YES & NO & Optional, on target & $\mathrm{O}$ & ${ }^{131} \mathrm{I}$ \\
\hline Hyperthyroidism nodules & ST & YES & NO & YES & NO & Optional, on target & $\mathrm{O}$ & ${ }^{131} \mathrm{I}$ \\
\hline non Hodgkin's lymphoma with 90Y Zevalin® & ST & NO & NO (5) & NO & NO & Non feasible with ${ }^{90} \mathrm{Y}$ & - & - \\
\hline 153Sm EDTMP Lexidronam (Quadramet@) & ST & YES & YES & YES & NO & RECOMMENDED on red marrow (2 WB counts) & $\mathrm{O}$ & ${ }^{153}$ Sm-EDTMP \\
\hline Radiosynoviorthesis & ST & NO & NO & NO & NO & Non feasible with ordinary methods & - & - \\
\hline Experimental therapies & NON ST & to be defined & YES & to be defined & YES & RECOMMENDED on OAR and TARGET. Methods to be defined & depends & depends \\
\hline PSMA & NON ST & YES & YES & YES & YES & $\begin{array}{l}\text { RECOMMENDED on OAR and target (predictive for the next treatment). } \\
\text { Methods to be defined }\end{array}$ & $\mathrm{O}$ & ${ }^{177}$ Lu labeled directed to PSMA \\
\hline
\end{tabular}

OAR $=$ organ at risk.

(1) Except particular cases described.

(2) Scout dose.

(3) Optional only if activity $<11$ GBq.

(4) Optional only if standard posology is used.

(5) No clinical need except rare cases.

$30 \%$ to $19 \%-24 \%$ ) and mIBG (from $67 \%$ to $43 \%$ to $22 \%$ ). In ${ }^{223} \mathrm{Ra}$, dosimetric optimization is absent in the data of 2015 and 2019 while dosimetric verification decreased in the three sequential surveys $(50 \%$ 14\%-5\%), but in 2015 and 2019 the number of centres performing it was constant (two).

\section{Discussion}

\section{Classification}

The two Associations believe that the classification of a therapy as standardized or non-standardized cannot derive from the rigid posology registration of the adopted radiopharmaceutical. The Italian classification was derived from the clinical complexity of patient/disease/therapy, where complexity is the uncertainty about outcome. Remnant ablation with fixed activity in DTC is effective in most cases, with absence of toxicity (apart from sporadic sialadenitis, still unpredictable with dosimetry). On the contrary, in the therapy of metastatic DTC, the outcome still spans from complete response to death. This easily lead to classify the former as standardized, while the latter as non-standardized. For Peptide Radio Receptor Therapy (PRRT) of Neuro-Endocrine Tumours (NET), similar variations in outcome are known [25]. In addition, the ${ }^{177} \mathrm{Lu}$-DOTATATE administration schedule is one among many possible [25]. Therefore, such therapy was classified as nonstandardized. Moreover, such schedule is nowadays under discussion, with the perspective of offering a prolonged patient benefit depending on individual tolerance $[6,15,21]$. Hepatic tumours treated with microspheres also result in a wide interval of overall survival, from complete response to early treatment related death [22]. Moreover, plenty of papers criticize the non-dosimetric methods indicated by manufacturers, in favour of tumour/non-tumour dosimetry, which is the basis for the optimization required by law $[6,15,21,26-31]$.

In summary, the primary therapy classification criterion should not be the drug registration availability, but the variability in efficacy and toxicity. In addition, methodological variations among centres could be considered. According to our criterion, two examples of standardized therapies are the treatment of benign thyroid diseases (curative therapy in most cases) and the therapy of bone metastases with ${ }^{223} \mathrm{Ra}$ (pure palliation intent). Their outcomes are opposite but definite. On the other side, the outcome of metastatic DTC patients spans from full recovery to death. Therefore, we classified such therapy as non-standardized. The classification as non-standardized therapy has the legal consequence of the close involvement of a medical physicist.

A common misinterpretation is that the classification as nonstandardized implies the recommendation of individualized dosimetric optimization and vice versa. The classification criterion and the optimization recommendation are neither unrelated, nor rigidly correlated concepts. Optimization is of course the ideal scenario for nonstandardized therapies, but this is not always recommended in Table 1, for technical difficulties or costs. Benign thyroid therapy is standardized, but optimization is recommended, to reduce stochastic risks in nononcological patients. As opposite example, mIBG treatment for adults is non-standardized, but optimization is indicated as optional in Table 1, mainly for cost/benefit reasons (see the supplementary materials). 


\section{Comparison with the EANM position paper about optimization of} NMT

The two Italian Associations recommend optimization in many more therapies than the European Association of Nuclear Medicine (EANM) [32]. EANM requires optimization, defined as the level 3 approach ("dosimetry-guided patient-specific prescription") only within research. Regarding treatments with approved agents in clinical routine, EANM does not exclude the use of dosimetry, but considers it advisable (level 2 or 3) in mIBG paediatric treatments. Furthermore, using approved agents, dosimetry is "required" only for ${ }^{166} \mathrm{Ho}$ microspheres, developed by a Dutch company, while for ${ }^{90} \mathrm{Y}$ microspheres is indicated as optional or advisable.

The two Associations tried to find a solution between the unequivocal, clear and transparent prescriptions of dosimetric optimization and verification by both the Directive and the Italian law, and the real situation, applying the argument about general cost and expected benefit from dosimetry [11]. Such expectation is not a chimera, as can be seen in detail in the supplementary materials. Plenty of evidence [33] of dose-effect relationships is available in radioiodine therapy of DTC [17,34-36], in PRRT of NET [37-39], and in liver radioembolization [22,40-44].

In addition to correlations between dose and effects, we do have indications of improved outcome thanks to the dosimetric approach. An increased objective response rate is reported in DTC radioiodine therapy $[12-14,45]$ while prolonged Progression Free Survival (PFS) and Overall Survival (OS) was observed in PRRT of neuroendocrine tumours [15], and in an outstanding multicentric prospective randomized study on hepatocarcinoma radioembolization (DOSISPHERE-01) [16].

Many renowned publications encourage individualized dosimetric optimization of nuclear medicine therapy.

About the indication of the EANM position paper of using patient cohort-averaged dosimetry in level 1 therapies, the International Commission on Radiological Protection (ICRP) itself in Publication 128 discourages such kind of approach: "The data are not intended for therapeutic applications of radionuclides. More detailed and patient-specific dosimetry and dose planning should be applied for therapeutic application of radionuclides." [46]

The International Agency for Atomic Energy (IAEA), in Specific Safety Guide No. 46, paragraph 4.812 states "Ideally, the administered activity should be based on the results of a pre-therapeutic dosimetry" and in paragraph 4.212 "Ideally, a pre-treatment calculation of the absorbed doses received by organs at risk and target tissues would allow for an accurate prediction of toxicity and efficacy of the treatment. The dosimetry calculations performed in this context should take into account individual patient pharmacokinetics and anatomy." [47]

The necessity of dosimetry to combine external beam and nuclear medicine therapy was reported by an eminent oncological journal [48]. NMT is named in that paper "Targeted Radio Therapy", TRT. "The design of treatment schedules that combine external beam radiotherapy and TRT remains a challenge, and the optimal treatment schedule, dosimetry, and radiobiology will probably differ for each vector-radionuclide pairing. A 2014 review found significant dose-response associations in 48 (61\%) of 79 studies of TRT [Strigari et al 33]. This finding emphasises the need for accurate dosimetry to achieve personalised administration for each TRT option."

\section{Italian surveys}

Survey data need some comment. We wrote that DTC remnant dosimetry is technically challenging using gammacamera. Some centres declared they performed it. At a phone investigation, they clarified that they measured only the uptake at $24 \mathrm{~h}$, assuming an average fixed effective half-life. Remnant volume was measured on scintigraphic images. This approach is affected by large uncertainties and it cannot be considered a real personalization.
The number of answering centres increased along time. This provided an augmented level of reliability especially in the two more recent surveys. Passing from 2015 to 2019, the percentual reduction of use of dosimetry in DTC, mIBG and ${ }^{223} \mathrm{Ra}$ is easily explained by a low but approximately constant number of centres performing dosimetry divided by a larger number of responding centres not performing dosimetry.

It is interesting that the use of dosimetry is more frequent in therapies (radioembolization, ${ }^{177}$ Lu-DOTATATE) introduced more recently than radioiodine in DTC and mIBG. More than half of the Italian centres perform dosimetry during ${ }^{177}$ Lu-DOTATATE therapy, where a real pretreatment evaluation with diagnostic activity is almost impossible, and where the rigid posology prevents a real optimization. Traditional applications (DTC, mIBG) evidently suffer from an inveterate habit to fixed activity administrations. This gap between new and old applications should be filled since the methodology is quite similar. Moreover, radioiodine instructions for use allow individualized therapy optimization with personalization of activity. In addition, the main pre-treatment dosimetry recommended to maximize therapy of metastatic DTC is the dosimetry to blood. This is quite feasible, being based on blood samples and whole-body counts. For therapeutic ${ }^{131} \mathrm{I}$-mIBG, IFU even suggests individualized dosimetric treatment planning (though with a too low maximum activity limit of 7.4 GBq).

The success of optimization in radioembolization may result from the minimum workload required for dosimetry (a single SPECT/CT scan ordinarily performed for clinical purpose), joined with the concern induced in therapist by the aggressiveness of liver disease and of this kind of therapy.

The interpretation of the 2019 survey data benefits from the attention to chronology. The Italian document was published in December 2018; the survey data refers to 2019 and the Italian law entered in force on 27 August 2020. We heard from some centre that the new national law will increase the rate of dosimetric approach with respect to the situation of 2019. Additional help in this trend derives from studies which demonstrated that acceptable dosimetric accuracy was obtained with only two [49] or even one scan [50] in ${ }^{177}$ Lu-DOTATATE, and only two scans with radioiodine [51]. We are therefore optimistic about further progress of optimization and dosimetry in Italy.

The most important remark regards the latest survey. ${ }^{177}$ Lu-DOTATATE and benign thyroid data demonstrate that clinical dosimetry is logistically feasible on a routine basis in Italy, not only with a single scan as in radioembolization, but also in systemic administrations requiring repeated scans. This situation will improve once the reimbursement for the practice of dosimetry will be available, as for all the others clinical practices.

\section{The supremacy of the radiation therapy legislation principles over the pharmacological principles}

The two Associations underline the fact that the criteria for prescribing the activity provided by some manufacturer based on fixed administered, or pro kilo, or based on criteria other than two compartment dosimetry to the target and to non-target tissues or organs, are in contrast with the requests of the Directive. Similar prescriptions clearly omit the application of the optimization principle. Such simplistic approaches can lead to damage or, at best, to lack of benefits potentially obtainable with an optimized and personalized administration. Although radio-pharmaceuticals are subject to pharmaceutical legislation, the therapeutic effect is mainly produced by the emission of ionizing radiation. Therefore, the two Associations state that these agents, as well as the medical devices used in NMT (radio-labelled microspheres), must first of all be subject to the legislation concerning radiotherapy, i.e. to article 56 of the Directive and to its translation into National laws in agreement with the optimization principle.

Based on this fundamental criterion (supremacy of the legislation concerning the aspects of radiation therapy over that relating to 
Table 3

Survey data about number of Italian centres performing dosimetry on a regular base.

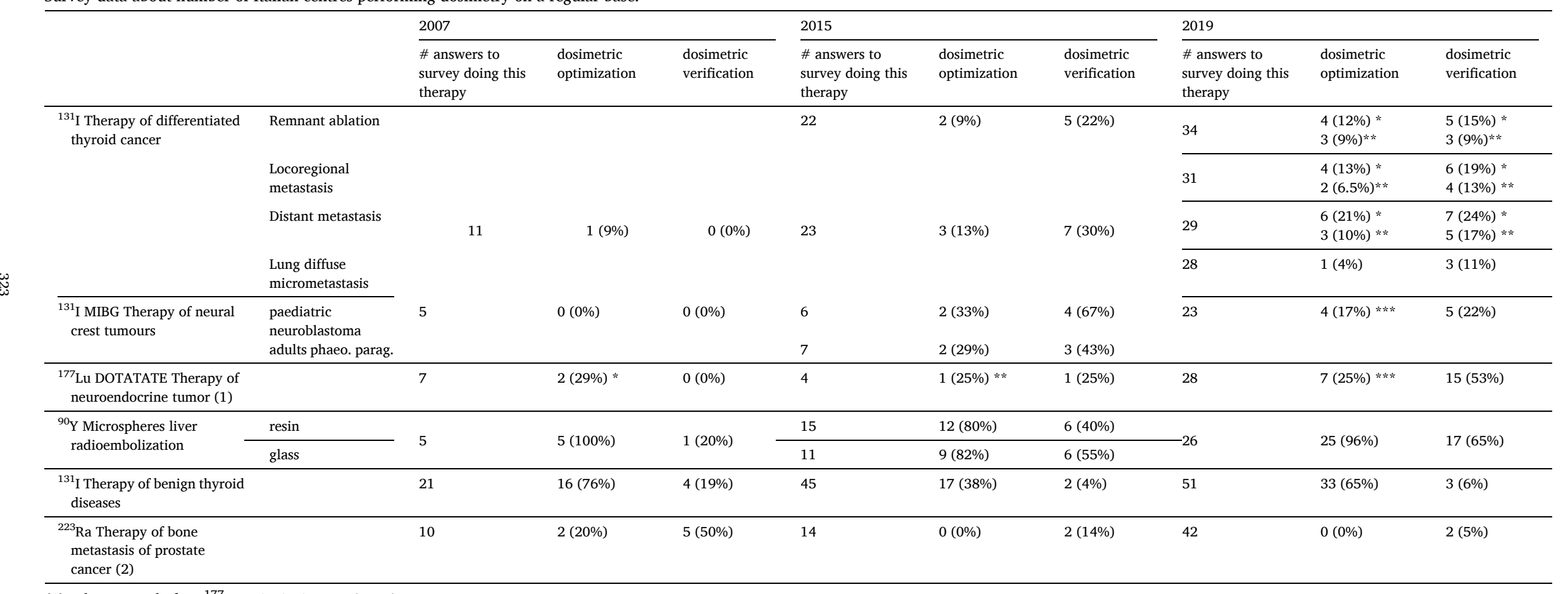

(1) other PRRT before ${ }^{177} \mathrm{Lu}$ DOTATATE registration.

(2) ${ }^{153} \mathrm{Sm}$-EDTMP before ${ }^{223} \mathrm{RaCl}_{2}$ registration.

(*) blood dosimetry.

$(* *)$ lesion dosimetry.

$(* * *)$ peri-therapy dosimetry to reduce the number of administrations. 
Italian Survey 2019

(61 responding hospitals)

\# answering centres doing this therapy $\quad$ \# dosimetric optimization $\quad$ \# dosimetric verification

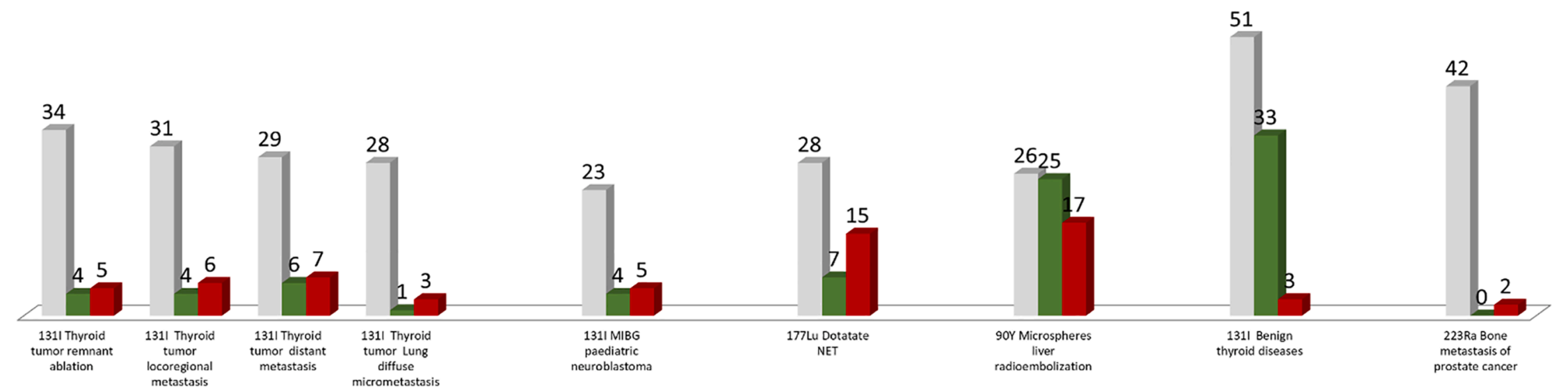

\section{EANM Survey 2015 (Italian data)}

(52 responding hospitals)

\# answering centres doing this therapy $\quad$ \# dosimetric optimization $\quad$ \# dosimetric verification

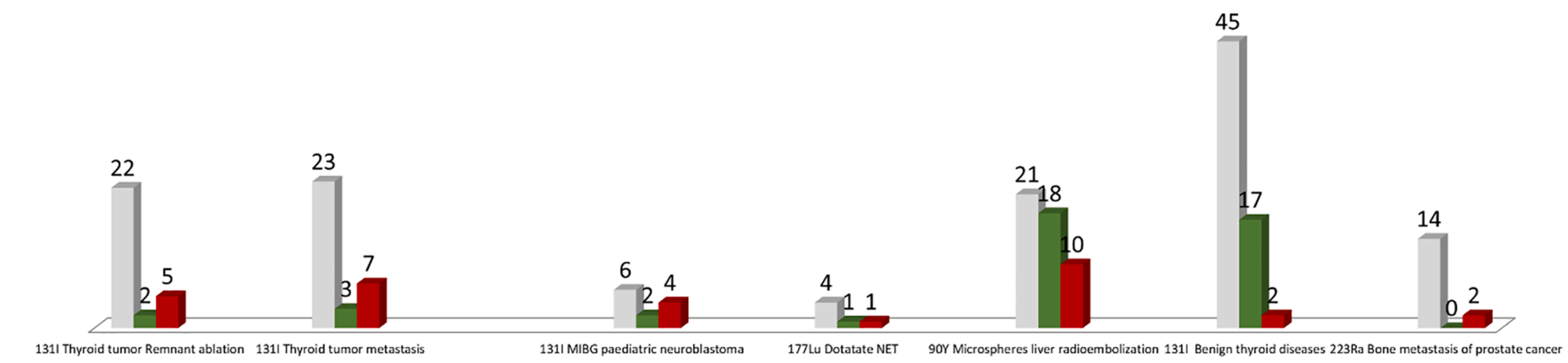

Fig. 1. Application of dosimetry in Italy according to the two major surveys about years 2015 [24] and 2019 [present work]. 
pharmaceuticals) the professionals responsible for the therapy should be legally allowed to optimize the treatment on a dosimetric basis with radiopharmaceuticals or medical devices approved, if necessary exceeding the posology of the IFU, under their own responsibility, without need of an approval by any ethics committee, without being within an experimental protocol. The therapeutic team should adopt published dose limit values to avoid toxicity to vital organs. In the absence of these dose limits (for instance renal tolerance with radio peptides labelled with ${ }^{177} \mathrm{Lu}$ ), the team must adopt published limits for similar therapies (renal tolerability with ${ }^{90} \mathrm{Y}$ radiopeptides), or, if they wish to optimize or maximize, undertake an experimental phase I study to determine these limits. The declarations of this paragraph and, in general, the whole present document have the explicit intent to act as legal support to optimization.

The two Associations encourage therapeutic teams to merge non dosimetric administration criteria with plenty of dose-effect correlation published data [33].

\section{Future perspectives: Resources}

The two Associations hope for Health Reimbursement Authorities (Regional Governments in Italy) will take on the optimization request of the Directive by identifying appropriate resources to implement it in NMT (man-time, scanner-time, software, radio-pharmaceuticals or medical devices).

The problem of economic resources (reimbursement) is not addressed in this document. It has to be differentiated according to the type of dosimetry. An additional distinction depends on whether dosimetry can be performed on an outpatient or inpatient basis.

\section{Conclusion: Formal appeal to regulatory agencies}

An editorial by European dosimetrists [6] had been sent before the registration of ${ }^{177}$ Lu-DOTATATE personally to the Director of European Medicines Agency (EMA) and to the responsible of the EMA Committee for Medicinal Products for Human Use. The aim was asking formal permission for a dosimetry-based optimization, in parallel with rigid posology. This had been completely disregarded.

The present document is another official formal appeal to EMA and to the Italian Agency for Pharmaceuticals (AIFA) in order to obtain explicit and formal acknowledgement of the legal possibility of performing NMT according to the optimization request of the Directive and of the Italian law, for agents approved in the past or to be approved in the future with rigid posology. In this historical phase, both administration criteria, approved rigid posology and personalized optimization, could be allowed in parallel. A similar situation already exists for instance for ${ }^{90} \mathrm{Y}$ resin microspheres, where activity can be chosen based on the non-dosimetric Body Surface Area approach, or on the tumour non tumour dosimetry (partition model). The same alternative is possible for radioiodine therapy of benign or oncological thyroid diseases, and for mIBG treatments. Optimized administration should not require an approval by any Ethics Committee, provided that dosimetric limits for OAR are respected. Such limits should be available in literature or deduced from phase I study of the same team previously approved by the Ethics Committee.

This appeal is itself a paradox since it is a request of permission of fulfilling the law (of radiotherapy). According to the EU Directive 2013/ 59 and the Italian law $101 / 2020$, the therapeutic team is subject to legal responsibility of optimization.

We presented the first consensus obtained between two National societies about the implementation of the optimization principle in NMT requested by the Directive. In the era of personalized and precision medicine and after the implementation in the member countries of a common legislation which gives maximal attention to patient dose both in diagnostic and in therapeutic practices, the two Associations trust in individualized optimization of nuclear medicine therapy where technically feasible and convenient. The decisions described in this document should not be considered valid everywhere and forever. They are rather pragmatic choices relative to Italy in the present situation. Scientific community should develop methodologies and science to improve treatment tailoring.

\section{Acknowledgments}

We are deeply in debt with Prof. Francesco Scopinaro, Sapienza University of Rome, who actively participated to the meetings for the development of the Italian version. He left this world before the development of this paper in English.

Dr. Gianluca Poli and Prof. Massimo Salvatori helped us with fruitful discussions.

We thank the two Italian Associations of Medical Physics and of Nuclear Medicine for the economic support to travel and meeting to develop this work. We are grateful to Dr. Silvia Ferrari who volunteered for the English language revision of the text.

\section{Appendix A. Supplementary data}

Supplementary data to this article can be found online at https://doi. org/10.1016/j.ejmp.2021.07.001.

\section{References}

[1] https://eur-lex.europa.eu/eli/dir/2013/59/oj.

[2] DECRETO LEGISLATIVO 31 luglio 2020, n. 101. Attuazione della direttiva 2013/ 59/Euratom, che stabilisce norme fondamentali di sicurezza relative alla protezione contro i pericoli derivanti dall'esposizione alle radiazioni ionizzanti, e che abroga le direttive 89/618/Euratom, 90/641/Euratom, 96/29/Euratom, 97/ 43/Euratom e 2003/122/Euratom e riordino della normativa di settore in attuazione dell'articolo 20, comma 1, lettera a), della legge 4 ottobre 2019, n. 117 . Supplemento ordinario alla "Gazzetta Ufficiale" n. 201 del 12 agosto 2020 - Serie generale.

[3] Chiesa C, Pacilio M, Strigari L, Bagni O, Maccauro M, Scopinaro F, Stasi M, Schillaci O. DOCUMENTO DI CONSENSUS INTERSOCIETARIO - TERAPIA MEDICO NUCLEARE: OTTIMIZZAZIONE SU BASE DOSIMETRICA AI SENSI DELLA DIRETTIVA EURATOM UE 2013/59 [https://www.fisicamedica.it/wp-content/up loads/Documenti/AIFM/Documenti\%20Intersocietari/DOCUMENTO\%20INTER SOCIETARIO\%20AIFM-AIMN\%20Finale.pdf].

[4] https://nedus.netkey.at/p/372/c/ee8c8ac308e318ae31da3108501a208b.

[5] Flux G, Bardies M, Chiesa C, Monsieurs M, Savolainen S, Strand S-E, et al. Clinical radionuclide therapy dosimetry: the quest for the "Holy Gray". Eur J Nucl Med Mol Imaging 2007;34(10):1699-700.

[6] Chiesa C, Sjogreen Gleisner K, Flux G, Gear J, Walrand S, Bacher K, et al. The conflict between treatment optimization and registration of radiopharmaceuticals with fixed activity posology in oncological nuclear medicine therapy. Eur J Nucl Med Mol Imaging 2017;44(11):1783-6.

[7] https://da4284glbbt4.cloudfront.net/Directives/MDD\%20Directive\%2093 42.pdf.

[8] www.btg-im.com/BTG/media/TheraSphere-Documents/PDF/10093509-Rev8_Eng lish-searchable.pdf.

[9] https://www.terumo-europe.com/en-emea/products/quiremspheres $\% \mathrm{C} 2 \%$ AE-microspheres.

[10] www.sirtex.com/media/155127/pi-ec-12.pdf.

[11] Eddy DM. Clinical decision making: from theory to practice. Cost-effectiveness analysis. Is it up to the task? JAMA 1992;267(24):3342-8.

[12] Lee JJ, Chung J-K, Kim SE, Kang WJ, Park DJ, Lee DS, et al. Maximal safe dose of I131 after failure of standard fixed dose therapy in patients with differentiated thyroid carcinoma. Ann Nucl Med 2008;22(9):727-34.

[13] Klubo-Gwiezdzinska J, Van Nostrand D, Atkins F, Burman K, Jonklaas J, Mete M, et al. Efficacy of dosimetric versus empiric prescribed activity of 131I for therapy of differentiated thyroid cancer. J Clin Endocrinol Metab 2011;96:3217-25.

[14] Dorn R, Kopp J, Vogt H, Heidenreich P, Carrol RG, Gulec SA. Dosimetry-guided radioactive iodine treatment in patients with metastatic differentiated thyroid cancer: largest safe dose using a risk-adapted approach. J Nucl Med 2003;44: 451-6.

[15] Garske-Román U, Sandström M, Fröss Baron K, Lundin L, Hellman P, Welin S, et al. Prospective observational study of 177Lu-DOTA-octreotate therapy in 200 patients with advanced metastasized neuroendocrine tumours (NETs): feasibility and impact of a dosimetry-guided study protocol on outcome and toxicity. Eur J Nucl Med Mol Imaging 2018;45(6):970-88.

[16] Garin E, Tselikas L, Guiu B, Chalaye J, Edeline J, de Baere T, et al. Personalised versus standard dosimetry approach of selective internal radiation therapy in patients with locally advanced hepatocellular carcinoma (DOSISPHERE-01): a randomised, multicentre, open-label phase 2 trial. Lancet Gastroenterol Hepatol 2021;6(1):17-29. 
[17] Maxon 3rd HR, Englaro EE, Thomas SR, Hertzberg VS, Hinnefeld JD, Chen LS, et al. Radioiodine-131 therapy for well-differentiated thyroid cancer-a quantitative radiation dosimetric approach: outcome and validation in 85 patients. J Nucl Med 1992;33:1132-6.

[18] Verburg FA, Luster M, Giovanella L, Lassmann M, Chiesa C, Chouin N, et al. Flux G for the EANM thyroid, radiation protection and dosimetry committees. The reset button revisited: why high activity 131I therapy of advanced differentiated thyroid cancer after dosimetry is advantageous for patients. Eur J Nucl Med Mol Imaging 2017;44(6):915-7.

[19] Benua RS, Cicale NR, Sonenberg M, Rawson RW, et al. The relation of radioiodine dosimetry to results and complications in the treatment of metastatic thyroid cancer. AJR 1962:171-82.

[20] Leeper RD. The effect of ${ }^{131}$ I therapy on survival of patients with metastatic papillary or folliculary thyroid carcinoma. J Clin Endocrinol Metab 1973;36: 1143-52.

[21] Sundlöv A, Sjögreen-Gleisner K, Svensson J, Ljungberg M, Olsson T, Bernhardt P, et al. Individualised ${ }^{17} 7 \mathrm{Lu}$-DOTATATE treatment of neuroendocrine tumours based on kidney dosimetry. Eur J Nucl Med Mol Imaging 2017;44(9):1480-9.

[22] Chiesa C, Mira M, Bhoori S, Bormolini G, Maccauro M, Spreafico C, et al. Radioembolization of hepatocarcinoma with ${ }^{90} \mathrm{Y}$ glass microspheres: treatment optimization using the dose-toxicity relationship. Eur J Nucl Med Mol Im 2020;47 (13):3018-32.

[23] Ahmad I, Nisar H. Dosimetry perspectives in radiation synovectomy. Phys Med 2018;47:64-72.

[24] Sjögreen Gleisner K, Spezi E, Solny P, Gabina PM, Cicone F, Stokke C, et al. Flux G Variations in the practice of molecular radiotherapy and implementation of dosimetry: results from a European survey. EJNMMI Phys 2017;4(1). https://doi. org/10.1186/s40658-017-0193-4.

[25] Zaknun JJ, Bodei L, Mueller-Brand J, Pavel ME, Baum RP, Hörsch D, et al. The joint IAEA, EANM, and SNMMI practical guidance on peptide receptor radionuclide therapy (PRRNT) in neuroendocrine tumours. Eur J Nucl Med Mol Imaging 2013; 40(5):800-16.

[26] Salem R, Padia SA, Lam M, Bell J, Chiesa C, Fowers K, et al. Clinical and dosimetric considerations for Y90: recommendations from an international multidisciplinary working group. Eur J Nucl Med Mol Imaging 2019;46(8):1695-704.

[27] Kafrouni M, Allimant C, Fourcade M, Vauclin S, Delicque J, Ilonca A-D, et al. FB retrospective voxel-based dosimetry for assessing the ability of the body-surfacearea model to predict delivered dose and radioembolization outcome. J Nucl Med 2018;59(8):1289-95.

[28] Kao YH, Hock Tan AE, Burgmans MC, Irani FG, Khoo LS, Gong Lo RH, et al. Imageguided personalized predictive dosimetry by artery-specific SPECT/CT partition modeling for safe and effective $90 Y$ radioembolization. J Nucl Med 2012;53(4): 559-66.

[29] Tong AKT, Kao YH, Too CW, Chin KFW, Ng DCE, Chow PKH. Chow PK Yttrium-90 hepatic radioembolization: clinical review and current techniques in interventional radiology and personalized dosimetry. Br J Radiol 2016;89(1062):20150943. https://doi.org/10.1259/bjr.20150943.

[30] Song YS, Paeng JC, Kim HC, Chung JW, Cheon GJ, Chung JK, Lee DS, Kang KW. PET/CT-Based Dosimetry in 90Y-Microsphere Selective Internal Radiation Therapy: Single Cohort Comparison With Pretreatment Planning on 99mTc-MAA Imaging and Correlation With Treatment Efficacy. Medicine 2015;94(23):1-8.

[31] Thomas MA, Mahvash A, Abdelsalam M, Kaseb AO, Kappadath SC. Planning dosimetry for ${ }^{90} \mathrm{Y}$ radioembolization with glass microspheres: Evaluating the fidelity of ${ }^{99} \mathrm{~m}$ Tc-MAA and partition model predictions. Med Phys 2020;47(10): $5333-42$.

[32] Konijnenberg M, Herrmann K, Kobe C, Verburg F, Hindorf C, Hustinx R, et al. EANM position paper on article 56 of the Council Directive 2013/59/Euratom (basic safety standards) for nuclear medicine therapy. Eur J Nucl Med Mol Imaging 2021;48(1):67-72.

[33] Strigari Lidia, Konijnenberg Mark, Chiesa Carlo, Bardies Manuel, Du Yong, Gleisner Katarina Sjögreen, et al. Flux G The evidence base for the use of internal dosimetry in the clinical practice of molecular radiotherapy. Eur J Nucl Med Mol Im 2014;41(10):1976-88.
[34] Maxon Harry R, Thomas Stephen R, Hertzberg Vicki S, Kereiakes James G, Chen IWen, Sperling Matthew I, et al. Relation between effective radiation dose and outcome of radioiodine therapy for thyroid cancer. N Engl J Med 1983;309(16): 937-41.

[35] Jentzen Walter, Hoppenbrouwers Jan, van Leeuwen Paul, van der Velden Daan, van de Kolk Rudie, Poeppel Thorsten Dirk, et al. Assessment of lesion response in the initial radioiodine treatment of differentiated thyroid cancer using 124I PET imaging. J Nucl Med 2014;55(11):1759-65.

[36] Jentzen Walter, Verschure Femke, van Zon Annelie, van de Kolk Rudie, Wierts Roel, Schmitz Jochen, et al. 124I PET assessment of response of bone metastases to initial radioiodine treatment of differentiated thyroid cancer. J Nucl Med 2016;57(10):1499-504.

[37] Wessels Barry W, Konijnenberg Mark W, Dale Roger G, Breitz Hazel B, Cremonesi Marta, Meredith Ruby F, et al. MIRD pamphlet No. 20: the effect of model assumptions on kidney dosimetry and response-implications for radionuclide therapy. J Nucl Med 2008;49(11):1884-99.

[38] Ilan Ezgi, Sandström Mattias, Wassberg Cecilia, Sundin Anders, Garske-Román Ulrike, Eriksson Barbro, et al. Dose response of pancreatic neuroendocrine tumors treated with peptide receptor radionuclide therapy using 177Lu-DOTATATE. J Nucl Med 2015;56(2):177-82.

[39] Cremonesi M, Ferrari ME, Bodei L, Chiesa C, Sarnelli A, Garibaldi C, et al. Correlation of dose with toxicity and tumour response to ${ }^{90} \mathrm{Y}$ - and ${ }^{177} \mathrm{Lu}$-PRRT provides the basis for optimization through individualized treatment planning. Eur J Nucl Med Mol Imaging 2018;45(13):2426-41.

[40] Cremonesi Marta, Chiesa Carlo, Strigari Lidia, Ferrari Mahila, Botta Francesca, Guerriero Francesco, et al. Radioembolization of hepatic lesions from a radiobiology and dosimetric perspective. Front Oncol 2014;4. https://doi.org/ 10.3389/fonc.2014.00210.

[41] Strigari L, Sciuto R, Rea S, Carpanese L, Pizzi G, Soriani A, et al. Efficacy and toxicity related to treatment of hepatocellular carcinoma with 90Y SIR spheres: radiobiological considerations. J Nucl Med 2010;51:1377-85.

[42] Lhommel Renaud, van Elmbt Larry, Goffette Pierre, Van den Eynde Marc, Jamar François, Pauwels Stanislas, et al. Feasibility of 90Y TOF PET-based dosimetry in liver metastasis therapy using SIR-Spheres. Eur J Nucl Med Mol Imaging 2010;37(9):1654-62.

[43] Flamen P, Vanderlinden B, Delatte P, et al CORRIGENDUM: Multimodality imaging can predict the metabolic response of unresectable colorectel liver metastases to radioembolization therapy with yttrium-90 labeled resin micropheres. Phys Med Biol 2014;59:2549.

[44] Lam Marnix GEH, Banerjee Arjun, Goris Michael L, Iagaru Andrei H, Mittra Erik S, Louie John D, et al. Fusion dual-tracer SPECT-based hepatic dosimetry predicts outcome after radioembolization for a wide range of tumour cell types. Eur J Nucl med Mol Im 2015;42(8):1192-201.

[45] Verburg FA. Advantages of dosimetry in 131I therapy of differentiated thyroid carcinoma. Quart J Nucl Med Mol Imaging 2019;63(3):253-7.

[46] Mattsson S, Johansson L, Leide Svegborn S, Liniecki J, Noßke D, Riklund KÅ, et al. ICRP PUBLICATION 128 radiation dose to patients from radiopharmaceuticals: a compendium of current information related to frequently used substances. Ann ICRP 2015;44(2_suppl):7-321.

[47] IAEA SAFETY STANDARDS SERIES No. SSG-46, Radiation Protection and Safety in Medical Uses of Ionizing Radiation https://www.iaea.org/publications/11102/ radiation-protection-and-safety-in-medical-uses-of-ionizing-radiation.

[48] Gill Martin R, Falzone Nadia, Du Yong, Vallis Katherine A. Targeted radionuclide therapy in combined-modality regimens. Lancet Oncol 2017;18(7):e414-23.

[49] Heikkonen Jorma, Mäenpää Hanna, Hippeläinen Eero, Reijonen Vappu, Tenhunen Mikko. Effect of calculation method on kidney dosimetry in 177Luoctreotate treatment. Acta Oncol 2016;55(9-10):1069-76.

[50] Hänscheid H, Lapa C, Buck AK, Lassmann M, Werner RA Dose Mapping After Endoradiotherapy with 177Lu-DOTATATE/DOTATOC by a Single Measurement After 4 Days J Nucl Med 2018 Jan;59(1):75-81.

[51] Jentzen Walter, Bockisch Andreas, Ruhlmann Marcus. Assessment of simplified blood dose protocols for the estimation of the maximum tolerable activity in thyroid cancer patients undergoing radioiodine therapy using 124I. J Nucl Med 2015;56(6):832-8. 